\title{
Announcements
}

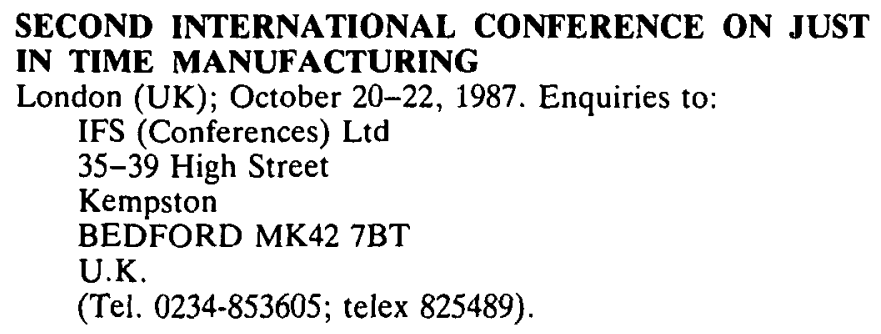

\section{SYMPOSIUM ON THE ART OF COMPUTER} CONVERSATION IN MEDICINE

Manchester (UK); October 22, 1987. Enquiries to:

Rosamund de Gama

Conference Division

BMIS

87 Gower Street

LONDON WC1E 6AA

U.K.

(Tel. 01-387-4949; telex 943763 Crocom G).

SEVENTEENTH INTERNATIONAL SYMPOSIUM ON COMPUTER INTEGRATED MANUFACTURING AND MANUFACTURING AUTOMATION PROTOCOL

Munich (FRG); October 25-29, 1987. Enquiries to:

ISATA Secretariat

42 Lloyd Park Avenue

CROYDON CR0 5SB

U.K.

(Tel. 01-680-8659; telex 28905 MONREF 2592).

\section{FIELD BUS SYMPOSIUM}

(Standards in the communication and process industries)

London (UK); November 3, 1987.

Enquiries to: Rosamund da Gama, as above.

SIXTH INTERNATIONAL CONFERENCE ON FMS

Turin (Italy); November 4-6, 1987.

Enquiries to: IFS, as above.

THIRD INTERNATIONAL CONFERENCE ON SIMULATION IN MANUFACTURING

Turin (Italy); November 4-6, 1987.

Enquiries to: IFS, as above.

\section{AN EXECUTIVE SEMINAR ON SUCCESSFUL AUTOMATED ASSEMBLY SYSTEMS London (UK); November 17-18, 1987.}

Enquiries to: IFS, as above.

\author{
MANAGEMENT CONFERENCE AND EXHIBITION \\ (BIM) \\ London (UK); November 16-17, 1987. Enquiries to: \\ B.I.M., Management House \\ Cottingham Road \\ CORBY \\ Northants NN17 1TT \\ U.K. \\ (Tel. 0536-204222).
}

\section{JIT EDUCATION AND IMPLEMENTATION} WORKSHOP... THE JOURNEY TO JUST IN TIME London (UK); November 17-19, 1987.

Enquiries to: IFS, as above

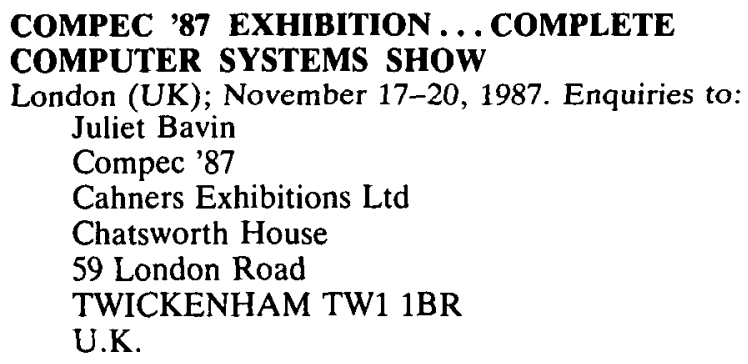

\section{CONFERENCE ON STATISTICAL PROCESS} CONTROL

Birmingham (UK); November 25-26, 1987.

Enquiries to: IFS, as above.

INTERNATIONAL CONGRESS ON COMMUNICATION AND COGNITION APPLIED EPISTEMOLOGY Ghent (Belgium); December 6-10, 1987. Enquiries to:

Communication and Cognition

Jan Van Dormael

Blandijnberg 2

B-9000 GHENT

Belgium

(Tel. 091-257571; telex 12754 Rugen).

INTERNATIONAL AND EXHIBITION... BUS, TRUCK \& CAR PRODUCT AND MANUFACTURING TECHNOLOGY (AUTOTECH '87)

Birmingham (UK); December 8-11, 1987. Enquiries to: AUTOTECH '87

National Exhibition Centre Ltd BIRMINGHAM B40 1NT

U.K.

(Tel. 021-7804171/4141; telex 336635 NECBHX). 
1987 WINTER SIMULATION CONFERENCE

Atlanta, Georgia (USA); December 14-16, 1987. Enquiries to:

Dr K.J. Musselman

Pritsker \& Associates, Inc.

P.O. Box 2413

WEST LAFAYETTE

IN 47906

USA

(Tel. 317-463-5557).

\section{SEVENTH INTERNATIONAL CONFERENCE ON} ROBOT VISION AND SENSORY CONTROLS

Zurich (Switzerland); February 2-4, 1988.

Enquiries to: IFS, as above.

\section{SCS MULTICONFERENCE}

San Diego, California (USA); February 3-5, 1988. Enquiries to:

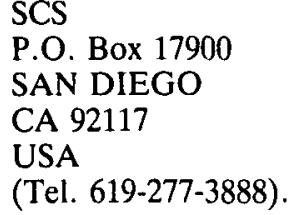

SCS

P.O. Box 17900

SAN DIEGO

CA 92117

USA

(Tel. 619-277-3888)

FIFTH WORLD WORLD INSTRUMENTATION AND INDUSTRIAL ELECTRONICS SYMPOSIUM AND EXHIBITION ... WISITEX ' 88

Bombay (India); February 4-10, 1988. Enquiries to: NOWEA INTERNATIONAL GmbH

Postfach 320203

Stockumer Kirchstrasse 61

D-4000 DÜSSELDORF 30

(Tel. 0211-456002; telex 8588351 now d).

\section{JIT EDUCATION AND IMPLEMENTATION WORKSHOP \\ Birmingham (UK); March 1-3, 1988:}

Enquiries to: IFS, as above.

LYON CONFERENCE ON MEDICAL IMAGING AND EXPERT SYSTEMS APPLIED TO MEDICINE

Lyon (France); March 10-12, 1988. Enquiries to: Package

Les Entretiens de Lyon, 55,

Montée de Choulans

69323 LYON Cedex 05

France

(Tel. 0337842 29 53; telex 330295 F).
INTERNATIONAL CONFERENCE ON EXTENDING DATABASE TECHNOLOGY

Venice (Italy); March 14-18, 1988. Enquiries to:

Michele Missikoff

IASA-CNR

Viale Manzoni 30, 00185

ROMA

Italy

(Tel. 396770031 ).

CONFERENCE ON COMPUTER METHODS AND

WATER RESOURCES IN DEVELOPING COUNTRIES

Rabat (Morocco); March 14-18, 1988. Enquiries to:

$\mathrm{CMI}$

52 Henstead Road

SOUTHAMPTON

SO1 2DD

U.K.

\section{NINTH INTERNATIONAL CONFERENCE ON ASSEMBLY AUTOMATION \\ London (UK); March 15-17, 1988.}

Enquiries to: IFS, as above.

CONFERENCE ON LOGISTICS (A.T. Kearney) London (UK); March 15-17, 1988.

Enquiries to: IFS, as above.

SIRI FOURTH INTERNATIONAL CONFERENCE ON ROBOTS BETWEEN SCIENCE AND TECHNOLOGY Milan (Italy); March 21-23, 1988. Enquiries to:

Segretaria Congresso SIRI presso CEU Spa

Viale Fulvio Testi 128

20092 Cinisello Balsamo

(MILANO)

Italy.

\section{CONFERENCE ON USER-ORIENTED CONTENT-} BASED TEXT AND IMAGE HANDLING

Cambridge, Massachusetts (USA); March 21-24, 1988. Enquiries to:

RIAO 88

CID

36 bis rue Ballu

75009 PARIS

France. 